\title{
On Ramanujan's cubic continued fraction
}

\author{
by \\ Heng Huat Chan (Urbana, Ill.) \\ Dedicated to the memory of Professor K. G. Ramanathan
}

\section{Introduction. Let}

$$
F(q)=\frac{q^{1 / 5}}{1}+\frac{q}{1}+\frac{q^{2}}{1}+\frac{q^{3}}{1}+\ldots, \quad|q|<1,
$$

denote the Rogers-Ramanujan continued fraction. On page 365 of his Lost Notebook [9], S. Ramanujan wrote five identities which show the relations between $F(q)$ and the five continued fractions $F(-q), F\left(q^{2}\right), F\left(q^{3}\right), F\left(q^{4}\right)$ and $F\left(q^{5}\right)([9]$, p. 365, (10), (11), (12), (13), (14)). An example of these identities is

$$
F^{5}(q)=F\left(q^{5}\right) \frac{1-2 F\left(q^{5}\right)+4 F^{2}\left(q^{5}\right)-3 F^{3}\left(q^{5}\right)+F^{4}\left(q^{5}\right)}{1+3 F\left(q^{5}\right)+4 F^{2}\left(q^{5}\right)+2 F^{3}\left(q^{5}\right)+F^{4}\left(q^{5}\right)},
$$

which shows a relation between $F(q)$ and $F\left(q^{5}\right)$. The proofs of (1.2) can be found in [10, p. 392, (7.1)], [7] and [11]. The proofs of those identities involving $F(-q), F\left(q^{2}\right), F\left(q^{3}\right)$ and $F\left(q^{4}\right)$ can be found in [1, p. 28], [1, p. 31], [10, p. 362, (6.2)] and [1, p. 34] respectively. For detailed discussions of (1.1), see [2, pp. 83-86], [7], [11] and [12].

On page 366 of his Lost Notebook [9], Ramanujan investigated another continued fraction

$$
G(q)=\frac{q^{1 / 3}}{1}+\frac{q+q^{2}}{1}+\frac{q^{2}+q^{4}}{1}+\frac{q^{3}+q^{6}}{1}+\ldots
$$

and claimed that there are many results of $G(q)$ which are analogous to those for (1.1). Motivated by Ramanujan's claim, we discovered many new identities which perhaps are the identities to which Ramanujan vaguely referred. In Section 2, we will establish the relations between $G(q)$ and the other three continued fractions $G(-q), G\left(q^{2}\right)$ and $G\left(q^{3}\right)$. These relations are analogous to those relations for $F(q)$ which are mentioned above. 
In his second letter to G. H. Hardy, Ramanujan claimed that

$$
\left(\frac{\sqrt{5}+1}{2}+F\left(e^{-2 \pi \alpha}\right)\right)\left(\frac{\sqrt{5}+1}{2}+F\left(e^{-2 \pi \beta}\right)\right)=\frac{5+\sqrt{5}}{2},
$$

where $\alpha \beta=1$. A companion identity of (1.4) is

$$
\left(\frac{\sqrt{5}-1}{2}-F\left(-e^{-\pi \alpha}\right)\right)\left(\frac{\sqrt{5}-1}{2}-F\left(-e^{-\pi \beta}\right)\right)=\frac{5-\sqrt{5}}{2} .
$$

Identities (1.4) and (1.5) were first proved by Watson [12]. Other proofs can be found in [2, p. 83] and [7]. In Section 3, we will prove some identities for $G(q)$ which are similar to (1.4) and (1.5).

In Section 4, we will apply the results of the previous two sections to compute some interesting numerical values of $G(q)$. In particular, we will give an analogue (see (4.3)) of Ramanujan's famous continued fraction

$$
\frac{e^{-2 \pi / 5}}{1}+\frac{e^{-2 \pi}}{1}+\frac{e^{-4 \pi}}{1}+\frac{e^{-6 \pi}}{1}+\ldots=\sqrt{\frac{5+\sqrt{5}}{2}}-\frac{\sqrt{5}+1}{2} .
$$

Identity (1.6) appeared in Ramanujan's first letter to Hardy and it is a direct consequence of (1.4) obtained by setting $\alpha=\beta=1$. In this section, we will also present a new algorithm for computing $\pi$.

On page 46 of his Lost Notebook [9], Ramanujan stated that

$$
\begin{array}{r}
\frac{e^{-\sqrt{3} \pi / 5}}{1}-\frac{e^{-\sqrt{3} \pi}}{1}+\frac{e^{-2 \sqrt{3} \pi}}{1}-\frac{e^{-3 \sqrt{3} \pi}}{1}+\ldots \\
=\frac{-(3+\sqrt{5})+\sqrt{6(5+\sqrt{5})}}{4} .
\end{array}
$$

The proof of (1.7) was first given by K. G. Ramanathan [8]. He proved (1.7) using Kronecker's Limit Formula and asserted that it was probably not Ramanujan's original proof. In Section 5, we will deduce (1.7) using a modular equation discovered by Ramanujan. We believe that we have rediscovered Ramanujan's proof. The use of modular equations in the computation of numerical continued fractions also provide an explanation for the presence of such complicated identities in Ramanujan's work. We will end this section with a computation of $G\left(-e^{-\sqrt{5} \pi}\right)$, which is an analogue of (1.7).

In the final section, we will show the equivalence of Borweins' cubic theta function identity and an identity for $G(q)$.

We end this introduction with definitions of certain functions which will be used in the sequel. We define

$$
(a ; q)_{\infty}:=\prod_{n=1}^{\infty}\left(1-a q^{n-1}\right), \quad|q|<1,
$$




$$
\begin{gathered}
f(-q):=(q ; q)_{\infty}, \\
\varphi(q):=\sum_{n=-\infty}^{\infty} q^{n^{2}}=\frac{(-q ;-q)_{\infty}}{(q ;-q)_{\infty}} \\
\psi(q):=f\left(q, q^{3}\right)=\sum_{n=0}^{\infty} q^{n(n+1) / 2}=\frac{\left(q^{2} ; q^{2}\right)_{\infty}}{\left(q ; q^{2}\right)_{\infty}}
\end{gathered}
$$

and

$$
\chi(-q)=\left(q ; q^{2}\right)_{\infty}
$$

\section{Statement and proof of Theorem 1}

Theorem 1. Let $G(q)$ be as defined in (1.3). Then

$$
\begin{gathered}
G(q)+G(-q)+2 G^{2}(-q) G^{2}(q)=0, \\
G^{2}(q)+2 G^{2}\left(q^{2}\right) G(q)-G\left(q^{2}\right)=0
\end{gathered}
$$

and

$$
G^{3}(q)=G\left(q^{3}\right) \frac{1-G\left(q^{3}\right)+G^{2}\left(q^{3}\right)}{1+2 G\left(q^{3}\right)+4 G^{2}\left(q^{3}\right)} .
$$

Proof of (2.1). Let $v=G(q)$ and $u=G(-q)$. (We will take $(-1)^{1 / 3}=$ -1.) From $[2$, p. 345, Entry 1(i)], $G(q)$ and $G(-q)$ have the evaluations

$$
v=q^{1 / 3} \frac{\chi(-q)}{\chi^{3}\left(-q^{3}\right)} \quad \text { and } \quad u=-q^{1 / 3} \frac{\chi(q)}{\chi^{3}\left(q^{3}\right)} .
$$

When

$q=\exp \left(-\pi \frac{{ }_{2} F_{1}\left(\frac{1}{2}, \frac{1}{2} ; 1 ; 1-\alpha\right)}{{ }_{2} F_{1}\left(\frac{1}{2}, \frac{1}{2} ; 1 ; \alpha\right)}\right)$ and $q^{3}=\exp \left(-\pi \frac{{ }_{2} F_{1}\left(\frac{1}{2}, \frac{1}{2} ; 1 ; 1-\beta\right)}{{ }_{2} F_{1}\left(\frac{1}{2}, \frac{1}{2} ; 1 ; \beta\right)}\right)$, we find that (see [2], p. 124, Entry 12(v), (vi))

$$
v=2^{-1 / 3} \frac{(1-\alpha)^{1 / 12} \beta^{1 / 8}}{(1-\beta)^{1 / 4} \alpha^{1 / 24}} \quad \text { and } \quad u=-2^{-1 / 3} \frac{(\beta(1-\beta))^{1 / 8}}{(\alpha(1-\alpha))^{1 / 24}} .
$$

Observe that

$$
\frac{u}{v}=-\left(\frac{(1-\beta)^{3}}{1-\alpha}\right)^{1 / 8} \text { and } \quad v u^{2}=\frac{1}{2}\left(\frac{\beta^{3}}{\alpha}\right)^{1 / 8} .
$$

From [2, p. 230, Entry 5(i)], we see that

$$
1=\left(\frac{(1-\beta)^{3}}{1-\alpha}\right)^{1 / 8}-\left(\frac{\beta^{3}}{\alpha}\right)^{1 / 8} .
$$

Hence, we have $-u / v-2 v u^{2}=1$. Simplifying, we obtain $v+u+2 v^{2} u^{2}=0$, which is (2.1). 
Proof of (2.2). From the identities given in (2.4), we compute

$$
\begin{aligned}
-G(q) G(-q) & =q^{2 / 3} \frac{\chi(-q) \chi(q)}{\chi^{3}\left(-q^{3}\right) \chi^{3}\left(q^{3}\right)} \\
& =q^{2 / 3} \frac{\chi\left(-q^{2}\right)}{\chi^{3}\left(-q^{6}\right)}=G\left(q^{2}\right) .
\end{aligned}
$$

If we multiply (2.1) by $G(q)$ and invoke (2.5), we obtain (2.2).

Proof of (2.3). Let $w:=G\left(q^{3}\right)$ and $v$ as defined in the proof of (2.1). From [2, p. 345, Entry 1(ii), (iv) and p. 347], we have

$$
\begin{gathered}
\frac{\varphi\left(-q^{3}\right)}{\varphi\left(-q^{1 / 3}\right)}=\frac{1}{1-2 v}, \\
3+\frac{f^{3}\left(-q^{1 / 3}\right)}{q^{1 / 3} f^{3}\left(-q^{3}\right)}=\frac{1}{v}+4 v^{2}=\left(27+\frac{f^{12}(-q)}{q f^{12}\left(-q^{3}\right)}\right)^{1 / 3}
\end{gathered}
$$

and

$$
\frac{\varphi^{4}(-q)}{\varphi^{4}\left(-q^{3}\right)}=1-8 v^{3},
$$

where $f(-q)$ and $\varphi(q)$ are the functions defined in (1.8) and (1.9). Using (2.6)-(2.8), with $q$ replaced by $q^{3}$ and $v$ replaced by $w$, we obtain

$$
\begin{aligned}
\frac{\varphi\left(-q^{9}\right)}{\varphi(-q)} & =\frac{1}{1-2 w} \\
3+\frac{f^{3}(-q)}{q f^{3}\left(-q^{9}\right)}=\frac{1}{w}+4 w^{2} & =\left(27+\frac{f^{12}\left(-q^{3}\right)}{q^{3} f^{12}\left(-q^{9}\right)}\right)^{1 / 3}
\end{aligned}
$$

and

$$
\frac{\varphi^{4}\left(-q^{3}\right)}{\varphi^{4}\left(-q^{9}\right)}=1-8 w^{3} .
$$

To prove (2.3), we also require the following identity [2, p. 345, Entry 1(iv)]:

$$
1+9 q \frac{f^{3}\left(-q^{9}\right)}{f^{3}(-q)}=\left(1+27 q \frac{f^{12}\left(-q^{3}\right)}{f^{12}(-q)}\right)^{1 / 3} .
$$

We first establish an identity that relates $v$ and $w$. Now, from the second equality of (2.7), we have

$$
\frac{1}{27}\left(\left(\frac{1}{v}+4 v^{2}\right)^{3}-27\right)=\frac{1}{27} \cdot \frac{f^{12}(-q)}{q f^{12}\left(-q^{3}\right)} .
$$

By (2.12) and (2.10), we obtain 


$$
\begin{aligned}
\frac{1}{27} \cdot \frac{f^{12}(-q)}{q f^{12}\left(-q^{3}\right)} & =\left(\left(1+9 q \frac{f^{3}\left(-q^{9}\right)}{f^{3}(-q)}\right)^{3}-1\right)^{-1} \\
& =\left(\left(1+9\left(\frac{1}{w}+4 w^{2}-3\right)^{-1}\right)^{3}-1\right)^{-1} \\
& =\frac{\left(1-3 w+4 w^{3}\right)^{3}}{\left(1+6 w+4 w^{3}\right)^{3}-\left(1-3 w+4 w^{3}\right)^{3}}
\end{aligned}
$$

Hence, we can deduce that

$$
\frac{1}{27}\left(\left(\frac{1}{v}+4 v^{2}\right)^{3}-27\right)=\frac{\left(1-3 w+4 w^{3}\right)^{3}}{\left(1+6 w+4 w^{3}\right)^{3}-\left(1-3 w+4 w^{3}\right)^{3}} .
$$

From (1.8)-(1.10) and Euler's identity, namely

we find that

$$
(-q ; q)_{\infty}=\frac{1}{\left(q ; q^{2}\right)_{\infty}},
$$

$$
\chi(-q)=\frac{\varphi(-q)}{f(-q)} .
$$

Using (2.4) and (2.16) we deduce that

$$
v=q^{1 / 3} \frac{\varphi(-q)}{f(-q)} \cdot \frac{f^{3}\left(-q^{3}\right)}{\varphi^{3}\left(-q^{3}\right)} \quad \text { and } \quad w=q \frac{\varphi\left(-q^{3}\right)}{f\left(-q^{3}\right)} \cdot \frac{f^{3}\left(-q^{9}\right)}{\varphi^{3}\left(-q^{9}\right)} .
$$

Thus,

$$
\frac{w}{v}=q^{2 / 3} \frac{\varphi^{4}\left(-q^{3}\right)}{\varphi^{4}\left(-q^{9}\right)} \cdot \frac{\varphi\left(-q^{9}\right)}{\varphi(-q)} \cdot \frac{f^{3}\left(-q^{9}\right)}{f^{3}(-q)} \cdot \frac{f^{4}(-q)}{f^{4}\left(-q^{3}\right)} .
$$

By (2.11), (2.9), the first equality of (2.10), and the second equality of (2.7), we have

$$
\begin{aligned}
\frac{w}{v}= & q^{2 / 3}\left(1+2 w+4 w^{2}\right)\left(q\left(\frac{1}{w}+4 w^{2}-3\right)\right)^{-1} \\
& \times q^{1 / 3}\left(\left(\frac{1}{v}+4 v^{2}\right)^{3}-27\right)^{1 / 3} \\
= & \frac{\left(1+2 w+4 w^{2}\right) w}{1+4 w^{3}-3 w}\left(\left(\frac{1}{v}+4 v^{2}\right)^{3}-27\right)^{1 / 3} .
\end{aligned}
$$

Finally, we cube both sides of (2.17) and use (2.15) to arrive at

$$
v^{3}=\frac{\left(1+6 w+4 w^{3}\right)^{3}-\left(1-3 w+4 w^{3}\right)^{3}}{\left(3\left(1+2 w+4 w^{2}\right)\right)^{3}} .
$$

Simplifying the right hand side of (2.18), we deduce (2.3).

Remark. The author would like to thank P. Borwein and B. Gordon for indicating the simplification of (2.18) which gives (2.3). 
3. Reciprocity theorems for $G(q)$

TheOrem 2. Let $\alpha \beta=1$. Then

$$
\begin{gathered}
\left(4 G^{2}\left(e^{-2 \pi \alpha}\right)+\frac{1}{G\left(e^{-2 \pi \alpha}\right)}-3\right) \\
\times\left(4 G^{2}\left(e^{-2 \pi \beta}\right)+\frac{1}{G\left(e^{-2 \pi \beta}\right)}-3\right)=27, \\
\left(1-2 G\left(-e^{-\pi \alpha}\right)\right)\left(1-2 G\left(-e^{-\pi \beta}\right)\right)=3,
\end{gathered}
$$

and

$$
\left(1-2 G\left(e^{-\sqrt{2} \pi \alpha}\right)\right)\left(1+\frac{1}{G\left(e^{-\sqrt{2} \pi \beta}\right)}\right)=3 .
$$

Pr o of of (3.1). We recall a transformation formula for $f(-q)[2$, p. 43 , Entry 27(iii)]:

$$
e^{-2 \pi z / 24} f\left(-e^{-2 \pi z}\right)=\frac{1}{\sqrt{z}} e^{-2 \pi /(24 z)} f\left(-e^{-2 \pi / z}\right) .
$$

From the first equality of (2.7), we observe that

$$
\begin{array}{r}
\left(4 G^{2}\left(e^{-2 \pi \alpha}\right)+\frac{1}{G\left(e^{-2 \pi \alpha}\right)}-3\right)\left(4 G^{2}\left(e^{-2 \pi \beta}\right)+\frac{1}{G\left(e^{-2 \pi \beta}\right)}-3\right) \\
=\frac{f^{3}\left(-e^{-2 \pi \alpha / 3}\right)}{e^{-2 \pi \alpha / 3} f^{3}\left(-e^{-6 \pi \alpha}\right)} \cdot \frac{f^{3}\left(-e^{-2 \pi \beta / 3}\right)}{e^{-2 \pi \beta / 3} f^{3}\left(-e^{-6 \pi \beta}\right)} .
\end{array}
$$

If we cube both sides of (3.4) and let $z=\alpha / 3$, we have

$$
e^{-\pi \alpha / 12} f^{3}\left(-e^{-2 \pi \alpha / 3}\right)=\left(\frac{3}{\alpha}\right)^{3 / 2} e^{-3 \pi \beta / 4} f^{3}\left(-e^{-6 \pi \beta}\right),
$$

since $\alpha \beta=1$. Similarly, with $z=\beta / 3$, we obtain

$$
e^{-\pi \beta / 12} f^{3}\left(-e^{-2 \pi \beta / 3}\right)=\left(\frac{3}{\beta}\right)^{3 / 2} e^{-3 \pi \alpha / 4} f^{3}\left(-e^{-6 \pi \alpha}\right) .
$$

Using (3.6) and (3.7), we can rewrite the right hand side of (3.5) as

$$
\frac{e^{\pi \alpha / 12} e^{-3 \pi \beta / 4}}{e^{-2 \pi \alpha / 3}}\left(\frac{3}{\alpha}\right)^{3 / 2} \frac{e^{\pi \beta / 12} e^{-3 \pi \alpha / 4}}{e^{-2 \pi \beta / 3}}\left(\frac{3}{\beta}\right)^{3 / 2}=27,
$$

as required.

Pro of of (3.2). We recall a transformation formula for $\varphi(q)[2$, p. 43, Entry 27(i)]:

$$
\varphi\left(e^{-\pi z}\right)=\frac{1}{\sqrt{z}} \varphi\left(e^{-\pi / z}\right), \quad \operatorname{Re} z>0
$$


From (2.6), we have

$$
\begin{aligned}
& \left(1-2 G\left(-e^{-\pi \alpha}\right)\right)\left(1-2 G\left(-e^{-\pi \beta}\right)\right) \\
& \quad=\frac{\varphi\left(e^{-\pi \alpha / 3}\right)}{\varphi\left(e^{-3 \pi \alpha}\right)} \cdot \frac{\varphi\left(e^{-\pi \beta / 3}\right)}{\varphi\left(e^{-3 \pi \beta}\right)}=\frac{\varphi\left(e^{-\pi \alpha / 3}\right)}{\varphi\left(e^{-3 \pi / \beta}\right)} \cdot \frac{\varphi\left(e^{-\pi \beta / 3}\right)}{\varphi\left(e^{-3 \pi / \alpha}\right)},
\end{aligned}
$$

since $\alpha \beta=1$. If we set $z=\beta / 3$ in (3.8), then

$$
\varphi\left(e^{-\beta \pi / 3}\right)=\sqrt{\frac{3}{\beta}} \varphi\left(e^{-3 \pi / \beta}\right) .
$$

Similarly, with $z=\alpha / 3$,

$$
\varphi\left(e^{-\alpha \pi / 3}\right)=\sqrt{\frac{3}{\alpha}} \varphi\left(e^{-3 \pi / \alpha}\right) .
$$

Using (3.10), (3.11) and $\alpha \beta=1$, we find from (3.9) that

$$
\left(1-2 G\left(-e^{-\pi \alpha}\right)\right)\left(1-2 G\left(-e^{-\pi \beta}\right)\right)=\sqrt{\frac{3}{\beta}} \sqrt{\frac{3}{\alpha}} \cdot \frac{\varphi\left(e^{-3 \pi / \alpha}\right)}{\varphi\left(e^{-3 \pi / \beta}\right)} \cdot \frac{\varphi\left(e^{-3 \pi / \beta}\right)}{\varphi\left(e^{-3 \pi / \alpha}\right)}=3,
$$

as required.

Proof of (3.3). We quote the following transformation formula [2, p. 43, Entry 27(ii)]:

$$
e^{-\pi z / 8} \psi\left(e^{-\pi z}\right)=\frac{1}{\sqrt{2 z}} \varphi\left(-e^{-2 \pi / z}\right),
$$

where $\psi(q)$ is the function defined in (1.10). We also require the identity [2, p. 345, Entry 1(i)]:

$$
1+\frac{1}{G(q)}=\frac{\psi\left(q^{1 / 3}\right)}{q^{1 / 3} \psi\left(q^{3}\right)}
$$

If we let $q=e^{-\sqrt{2} \pi \alpha}$ in (3.13) and invoke (3.12), we obtain

$$
1+\frac{1}{G\left(e^{-\sqrt{2} \pi \alpha}\right)}=\frac{\psi\left(e^{-\sqrt{2} \pi \alpha / 3}\right)}{e^{-\sqrt{2} \pi \alpha / 3} \psi\left(e^{-3 \sqrt{2} \pi \alpha}\right)}=3 \frac{\varphi\left(-e^{-3 \sqrt{2} \pi / \alpha}\right)}{\varphi\left(-e^{-\sqrt{2} \pi /(3 \alpha)}\right)} .
$$

On the other hand, by (2.6) with $q=e^{-\sqrt{2} \pi / \alpha}$, we deduce that

$$
\frac{\varphi\left(-e^{-3 \sqrt{2} \pi / \alpha}\right)}{\varphi\left(-e^{-\sqrt{2} \pi /(3 \alpha)}\right)}=\frac{1}{1-2 G\left(e^{-\sqrt{2} \pi / \alpha}\right)}=\frac{1}{1-2 G\left(e^{-\sqrt{2} \pi \beta}\right)},
$$

since $\alpha \beta=1$. Combining (3.14) and (3.15), we conclude our proof of (3.3). 
4. Some numerical values of $G(q)$ and an algorithm for computing $\pi$

Theorem 3.

$$
\begin{gathered}
G\left(-e^{-\pi}\right)=\frac{1-\sqrt{3}}{2}, \\
G\left(e^{-\pi}\right)=\frac{(1+\sqrt{3})(-(1+\sqrt{3})+\sqrt{6 \sqrt{3}})}{4}, \\
G\left(e^{-2 \pi}\right)=\frac{-(1+\sqrt{3})+\sqrt{6 \sqrt{3}}}{4}, \\
G\left(e^{-\sqrt{2} \pi}\right)=\frac{-2+\sqrt{6}}{2}, \\
G^{3}\left(e^{-\sqrt{2} \pi / 3}\right)=\frac{G\left(e^{-\sqrt{2} \pi}\right)}{2} .
\end{gathered}
$$

Proof of (4.1). If $\alpha=\beta=1$ in (3.2), then

$$
\left(1-2 G\left(-e^{-\pi}\right)\right)^{2}=3 \text {, }
$$

and this proves (4.1)

Pro of of (4.2). To evaluate $G\left(e^{-\pi}\right)$, we solve the quadratic equation

$$
2 G^{2}\left(-e^{-\pi}\right) x^{2}+x+G\left(-e^{-\pi}\right)=0,
$$

which is obtained from (2.1).

Proof of (4.3). Using (2.5), (4.1) and (4.2), we deduce that

$$
G\left(e^{-2 \pi}\right)=-G\left(-e^{-\pi}\right) G\left(e^{-\pi}\right)=\frac{-(1+\sqrt{3})+\sqrt{6 \sqrt{3}}}{4} .
$$

Proof of (4.4). For simplicity, we let $A:=G\left(e^{-\sqrt{2} \pi}\right)$. If we set $\alpha=$ $\beta=1$ in (3.3), then we obtain

$$
(A+1)(2 A-1)=-3 A .
$$

Solving (4.6) yields (4.4).

Pr o of of (4.5). We substitute (4.4) into (2.3) to obtain (4.5).

We will end this section with a new algorithm for computing $\pi$.

TheOrem 4. Let

$$
A_{1}=\frac{-(1+\sqrt{3})+\sqrt{6 \sqrt{3}}}{4}, \quad A_{n}=\frac{2 A_{n-1}^{2}}{1+\sqrt{1-8 A_{n-1}^{3}}}, \quad n>1 .
$$

Then

$$
\lim _{n \rightarrow \infty}-3 \frac{\ln A_{n}}{2^{n}}=\pi
$$


Pr o of. Let $B_{n}:=G\left(\exp \left(-2^{n} \pi\right)\right)$. Then by (2.2) and (4.3), we have

$$
B_{1}=\frac{-(1+\sqrt{3})+\sqrt{6 \sqrt{3}}}{4}=A_{1}
$$

and

$$
B_{n}=\frac{1-\sqrt{1-8 A_{n-1}^{3}}}{4 A_{n-1}}=\frac{2 A_{n-1}^{2}}{1+\sqrt{1-8 A_{n-1}^{3}}}=A_{n}, \quad n>1 .
$$

Since $G(q)=q^{1 / 3}(1+O(q))$, we have

$$
A_{n} \sim \exp \left(\frac{-2^{n} \pi}{3}\right) \quad \text { as } n \rightarrow \infty .
$$

Hence the assertion follows.

Remarks. Using Mathematica and the algorithm given in Theorem 4, we are able to verify that our approximation agrees with $\pi$ up to 10,22 , $42,88,175,350$, and 701 decimal places when $n=3,4,5,6,7,8$, and 9 , respectively. The computation of the logarithm is non-trivial. One can get rid of the logarithm and obtain an efficient algorithm for computing $e^{\pi}$. For more efficient algorithms for computing $\pi$, see [4].

5. Modular equations and Ramanujan's continued fractions. In this section, we will present a new proof of (1.7) and a computation of $G\left(e^{-\sqrt{5} \pi}\right)$.

To prove (1.7), we recall a transformation formula for $f(q)[2$, p. 43 , Entry 27(iv)]:

$$
e^{-\pi z / 24} f\left(e^{-\pi z}\right)=\frac{1}{\sqrt{z}} e^{-\pi /(24 z)} f\left(e^{-\pi / z}\right) .
$$

We also require the following modular equation.

LEMma 1. Let

$$
P=\frac{f(q)}{q^{1 / 12} f\left(q^{3}\right)} \quad \text { and } \quad Q=\frac{f\left(q^{5}\right)}{q^{5 / 12} f\left(q^{15}\right)} .
$$

Then

$$
(P Q)^{2}+\frac{9}{(P Q)^{2}}=\left(\frac{Q}{P}\right)^{3}-\left(\frac{P}{Q}\right)^{3}+5 .
$$

For a proof of Lemma 1, see [3, Chapter 25, Entry 62].

Let $q=e^{-\pi / \sqrt{3}}$ in Lemma 1. By (5.1), we deduce that

$$
P=\frac{f\left(e^{-\pi / \sqrt{3}}\right)}{e^{-\pi /(12 \sqrt{3})} f\left(e^{-\sqrt{3} \pi}\right)}=3^{1 / 4} .
$$


Similarly,

$$
Q=\frac{f\left(e^{-5 \pi / \sqrt{3}}\right)}{e^{-5 \pi /(12 \sqrt{3})} f\left(e^{-5 \sqrt{3} \pi}\right)}=\frac{3^{1 / 4}}{\sqrt{5}} e^{\sqrt{3} \pi / 5} \frac{f\left(e^{-\sqrt{3} \pi / 5}\right)}{f\left(e^{-5 \sqrt{3} \pi}\right)} .
$$

If we let

$$
B:=e^{\sqrt{3} \pi / 5} \frac{f\left(e^{-\sqrt{3} \pi / 5}\right)}{f\left(e^{-5 \sqrt{3} \pi}\right)},
$$

then

$$
P Q=\sqrt{\frac{3}{5}} B \text { and } \quad \frac{Q}{P}=\frac{B}{\sqrt{5}} .
$$

Substituting (5.3) into Lemma 1, we obtain

$$
\frac{3}{5} B^{2}+\frac{15}{B^{2}}=\left(\frac{B}{\sqrt{5}}\right)^{3}-\left(\frac{\sqrt{5}}{B}\right)^{3}+5
$$

We may rewrite (5.4) as

$$
3\left(\left(\frac{B}{\sqrt{5}}-\frac{\sqrt{5}}{B}\right)^{2}+2\right)=\left(\frac{B}{\sqrt{5}}-\frac{\sqrt{5}}{B}\right)^{3}+3\left(\frac{B}{\sqrt{5}}-\frac{\sqrt{5}}{B}\right)+5,
$$

which implies that

$$
\left(\left(\frac{B}{\sqrt{5}}-\frac{\sqrt{5}}{B}\right)-1\right)^{3}=0
$$

Solving (5.5), we obtain

$$
B=(5+\sqrt{5}) / 2 .
$$

Now, from $[2$, p. $84,(39.1)]$, we know that

$$
\frac{-1}{F\left(-e^{-\sqrt{3} \pi}\right)}+F\left(-e^{-\sqrt{3} \pi}\right)+1=e^{\sqrt{3} \pi / 5} \frac{f\left(e^{-\sqrt{3} \pi / 5}\right)}{f\left(e^{-5 \sqrt{3} \pi}\right)} .
$$

By (5.2) and (5.6), we conclude that the right hand side of (5.7) equals $(5+\sqrt{5}) / 2$. Solving the quadratic equation (5.7), we obtain (1.7).

Remark. In (1.7), $F(q)$ is evaluated at $q=-e^{-\sqrt{3} \pi}$. An analogous result for $G(q)$ would be its value at $q=-e^{-\sqrt{5} \pi}$.

Next, we state and prove

\section{THEOREM 5.}

$$
G\left(-e^{-\sqrt{5} \pi}\right)=\frac{(\sqrt{5}-3)(\sqrt{5}-\sqrt{3})}{4} .
$$

To prove (5.8), we require the following identity. 
Lemma 2. Let $P=\varphi(q) / \varphi\left(q^{5}\right)$ and $Q=\varphi\left(q^{3}\right) / \varphi\left(q^{15}\right)$. Then

$$
P Q+\frac{5}{P Q}=\left(\frac{Q}{P}\right)^{2}+3 \frac{Q}{P}+3 \frac{P}{Q}-\left(\frac{P}{Q}\right)^{2} .
$$

For a proof of Lemma 2, see [3, Chapter 25, Entry 67].

Proof of Theorem 5 . We let $q=e^{-\pi / \sqrt{5}}$ and invoke (3.8) to deduce that

$$
P=\frac{\varphi\left(e^{-\pi / \sqrt{5}}\right)}{\varphi\left(e^{-\sqrt{5} \pi}\right)}=5^{1 / 4}, \quad Q=\frac{\varphi\left(e^{-3 \pi / \sqrt{5}}\right)}{\varphi\left(e^{-3 \sqrt{5} \pi}\right)}=\frac{5^{1 / 4}}{\sqrt{3}} \cdot \frac{\varphi\left(e^{-\sqrt{5} \pi / 3}\right)}{\varphi\left(e^{-3 \sqrt{5} \pi}\right)} .
$$

If we let

$$
C:=\frac{\varphi\left(e^{-\sqrt{5} \pi / 3}\right)}{\varphi\left(e^{-3 \sqrt{5} \pi}\right)}
$$

then

$$
P Q=\sqrt{\frac{5}{3}} C \text { and } \frac{P}{Q}=\frac{\sqrt{3}}{C} .
$$

Substituting (5.10) into Lemma 2, we obtain

$$
\sqrt{\frac{5}{3}} C+\frac{\sqrt{15}}{C}=\left(\frac{C}{\sqrt{3}}\right)^{2}+3 \frac{C}{\sqrt{3}}+3 \frac{\sqrt{3}}{C}-\left(\frac{\sqrt{3}}{C}\right)^{2}
$$

which may be rewritten as

$$
\sqrt{5}\left(\frac{C}{\sqrt{3}}+\frac{\sqrt{3}}{C}\right)=\left(\frac{C}{\sqrt{3}}-\frac{\sqrt{3}}{C}\right)\left(\frac{C}{\sqrt{3}}+\frac{\sqrt{3}}{C}\right)+3\left(\frac{C}{\sqrt{3}}+\frac{\sqrt{3}}{C}\right) .
$$

Since $C / \sqrt{3}+\sqrt{3} / C \neq 0$, we have

$$
\sqrt{5}=\left(\frac{C}{\sqrt{3}}-\frac{\sqrt{3}}{C}\right)+3
$$

Solving the quadratic equation (5.11), we find that

$$
C=(-3 \sqrt{3}+\sqrt{15}+3(\sqrt{5}-1)) / 2 .
$$

From (2.6), we know that

$$
1-2 G\left(-e^{-\sqrt{5} \pi}\right)=\frac{\varphi\left(e^{-\sqrt{5} \pi / 3}\right)}{\varphi\left(e^{-3 \sqrt{5} \pi}\right)}
$$

Thus, (5.8) follows from (5.9), (5.12) and (5.13).

Remark. A completely different proof of (5.12) can be found in $[2$, p. $210,(23.5)]$. 
6. A proof of the Borweins' cubic theta function identity. We first define

$$
a(q)=\sum_{m, n=-\infty}^{\infty} q^{m^{2}+m n+n^{2}}, \quad b(q)=\sum_{m, n=-\infty}^{\infty} \omega^{n-m} q^{n^{2}+m n+m^{2}}
$$

and

$$
c(q)=\sum_{m, n=-\infty}^{\infty} q^{(n+1 / 3)^{2}+(n+1 / 3)(m+1 / 3)+(m+1 / 3)^{2}},
$$

where $\omega$ is a primitive cube root of unity. In [5], P. Borwein and J. Borwein showed that

$$
a^{3}(q)=b^{3}(q)+c^{3}(q) .
$$

Furthermore, the Borweins and F. Garvan [6] proved that

$$
a(q)=\frac{f^{3}\left(-q^{1 / 3}\right)+3 q^{1 / 3} f^{3}\left(-q^{3}\right)}{f(-q)}, \quad b(q)=\frac{f^{3}(-q)}{f\left(-q^{3}\right)}
$$

and

$$
c(q)=3 q^{1 / 3} \frac{f^{3}\left(-q^{3}\right)}{f(-q)} .
$$

From the above theta function representations of $a(q), b(q)$ and $c(q)$, we note that in order to prove (6.1), it suffices to show that

$$
\left(\frac{f^{3}\left(-q^{1 / 3}\right)+3 q^{1 / 3} f^{3}\left(-q^{3}\right)}{f(-q)}\right)^{3}=\left(\frac{f^{3}(-q)}{f\left(-q^{3}\right)}\right)^{3}+\left(3 q^{1 / 3} \frac{f^{3}\left(-q^{3}\right)}{f(-q)}\right)^{3}
$$

If we multiply both sides of $(6.2)$ by $\left(\frac{f(-q)}{q^{1 / 3} f^{3}\left(-q^{3}\right)}\right)^{3}$, we obtain

$$
\left(\frac{f^{3}\left(-q^{1 / 3}\right)}{q^{1 / 3} f^{3}\left(-q^{3}\right)}+3\right)^{3}=\frac{f^{12}(-q)}{q f^{12}\left(-q^{3}\right)}+27,
$$

which is identity $(2.7)$.

Remark. One can deduce (2.7) from (6.1). For more details, see [6, Corollary 2.5].

Acknowledgements. The author would like to thank B. C. Berndt for bringing his attention to [9, pp. 364-366].

\section{References}

[1] G. E. Andrews, B. C. Berndt, L. Jacobsen and R. C. Lamphere, The continued fractions found in the unorganised portions of Ramanujan's notebooks, Mem. Amer. Math. Soc. 477 (1992).

[2] B. C. Berndt, Ramanujan Notebooks, Part III, Springer, New York, 1991. 
[3] B. C. Berndt, Ramanujan Notebooks, Part IV, Springer, New York, 1994.

[4] J. M. Borwein and P. B. Borwein, Pi and the AGM, Wiley, New York, 1987.

[5] - - - A cubic counterpart of Jacobi's identity and the AGM, Trans. Amer. Math. Soc. 323 (1991), 691-701.

[6] J. M. Borwein, P. B. Borwein and F. G. Garvan, Some cubic identities of Ramanujan, ibid. 343 (1994), 35-47.

[7] K. G. Ramanathan, On Ramanujan's continued fraction, Acta Arith. 43 (1984), 209-226.

[8] - On some theorems stated by Ramanujan, in: Number Theory and Related Topics, Tata Inst. Fund. Res. Stud. Math. 12, Oxford University Press, Bombay, 1989, 151160 .

[9] S. Ramanujan, The Lost Notebook and Other Unpublished Papers, Narosa, New Delhi, 1988.

[10] L. J. Rogers, On a type of modular relation, Proc. London Math. Soc. 19 (1921), 387-397.

[11] G. N. Watson, Theorems stated by Ramanujan (VII): theorems on continued fractions, J. London Math. Soc. 4 (1929), 39-48.

[12] - Theorems stated by Ramanujan (IX): two continued fractions, ibid., 231-237.

DEPARTMENT OF MATHEMATICS

UNIVERSITY OF ILLINOIS

273 ALTGELD HALL

1409 WEST GREEN STREET

URBANA, ILLINOIS 61801

U.S.A. 